\title{
Comparison of the main components and bioactivity of Rhus verniciflua Stokes extracts by different detoxification processing methods
}

\author{
Seon-Ok Lee ${ }^{1}$, Sung-Ji Kim ${ }^{2}$, Ju-Sung Kim ${ }^{4}$, Hyuk Ji ${ }^{1}$, Eun-Ok Lee ${ }^{1,2,3}$ and Hyo-Jeong Lee ${ }^{1,2,3^{*}}$
}

\begin{abstract}
Background: Rhus verniciflua Stokes is an Asian tree species that is used as a food supplement and traditional medicine in Korea. However, its use is restricted by its potential to cause allergy. Thus, allergen-free $R$. verniciflua extracts are currently being marketed as a functional health food in Korea. In the present study, three different allergen-free $R$. verniciflua extracts (DRVE, FRVE, and FFRVE) were produced by detoxification of $R$. verniciflua, and their properties and constituents were compared.
\end{abstract}

Methods: The main components and properties (antibacterial, antioxidant, anticancer, and hepatic lipogenesis inhibitory effects) of the three allergen-free extracts were compared. Moreover, the major phenolic constituents of $R$. verniciflua, including gallic acid, fustin, fisetin, and quercetin, were analyzed in the three extracts.

Results: DRVE was superior to the two other extracts with regard to antioxidant activity, while FRVE was superior with regard to antimicrobial activity and suppression of hepatic lipogenesis. FRVE exhibited lipid-lowering effects by lowering sterol regulatory element-binding protein 1 and triglyceride levels, and promoting the activation of peroxisome proliferator-activated receptor and AMP-activated protein kinase in an in vitro model of non-alcoholic fatty liver.

Conclusions: Overall, our findings demonstrate various differences among the three extracts. This suggests that functional and bioactive compounds present in R. verniciflua could be altered by the detoxification process, and this property could be considered in the development of functional health foods in the future.

Keywords: Rhus verniciflua Stokes, Detoxification, Fermentation, Fustin, Fisetin

\section{Background}

Rhus verniciflua Stokes (RVS), the lacquer tree, has been used as a traditional medicine and food supplement for a long time in Eastern Asia. In Korea, RVS has been used as a herbal medicine for the treatment of abdominal pain, including pain caused by stomach disorders such as gastritis, and as a hemostatic agent [1]. RVS has been reported to exhibit anticancer, antioxidant, antimicrobial, and anti-

\footnotetext{
* Correspondence: strong79@khu.ac.kr

${ }^{1}$ Department of Science in Korean Medicine, Graduate School, Kyung Hee University, Hoegi-dong, Dongdaemun-gu, 130-701 Seoul, Republic of Korea 2Department of Cancer Preventive Material Development, Graduate School, Kyung Hee University, Hoegi-dong, Dongdaemun-gu, 130-701 Seoul, Republic of Korea

Full list of author information is available at the end of the article
}

inflammatory activities [2-6]. Moreover, RVS contains a wide variety of flavonoids and polyphenols, including fustin, fisetin, quercetin, butein, p-coumaric acid, kaempferol, sulfuretin, catechol, and ethyl gallate. However, despite the various biological activities of RVS, its use has been limited because of a component called urushiol, which is known to cause allergies. Therefore, urushiol should be removed before using RVS as a food supplement or medicine. Several detoxification methods have been developed to produce urushiol-free RVS, such as heat treatment, solvent extraction, and enzyme treatment by microbial or mushroom mycelium fermentation [7-9]. Currently, allergen-free RVS extracts are being marketed as health functional food in Korea. However, there are no comparative studies on the

(c) The Author(s). 2018 Open Access This article is distributed under the terms of the Creative Commons Attribution 4.0 International License (http://creativecommons.org/licenses/by/4.0/) which permits unrestricted use, distribution, and reproduction in any medium, provided you give appropriate credit to the original author(s) and the source, provide a link to the Creative Commons license, and indicate if changes were made. The Creative Commons Public Domain Dedication waiver (http://creativecommons.org/publicdomain/zero/1.0/) applies to the data made available in this article, unless otherwise stated. 
components and bioactivities of RVS extracts obtained by various detoxification methods. It is important to develop efficient and cost-effective food processing methods in order to enhance the content of bioactive components.

We previously reported that RVS detoxified via a microbial method could alleviate oleic acid (OA)-induced steatosis in HepG2 cells, and that it contained phenolics and cosanols with lipid-lowering potential. In the present study, we compared the effects of three RVS extracts detoxified by different methods with regard to their antioxidant, antimicrobial, and anticancer properties, their suppressive effect on hepatic lipogenesis, and their main components.

\section{Methods}

\section{Preparation of DRVE, FRVE, and FFRVE powders}

The three types of detoxified RVS extracts used in this study are products that are commercially available in Korea, and were purchased from Okkane (Seoul, Korea). The following three extracts were used: an allergen-free RVS extract detoxified by hot air drying (dried $R$. verniciflua extract, DRVE), an allergen-free RVS extract fermented with Saccharomyces carlsbergensis (fermented $R$. verniciflua extract, FRVE), and an allergen-free RVS extract fermented with mushroom mycelium (Fomitella fraxinea-fermented $R$. verniciflua extract, FFRVE). The extracts (one bottle $=1.5 \mathrm{~L}$ ) were dried to a powder by freeze-drying.

\section{Determination of antimicrobial activity by agar diffusion method}

The antimicrobial activities of the three RVS extracts were determined via modified Kirby-Bauer disk diffusion method $[10,11]$. The test microorganisms used in this experiment were Propionibacterium acnes (ATCC 6919) and Trichophyton rubrum (ATCC 22402), and were obtained from the Korean Culture Center of Microorganisms. $P$. acnes was cultured anaerobically at $37{ }^{\circ} \mathrm{C}$ in Mueller Hinton broth (Difco, USA) and Mueller Hinton agar $\left(0.75 \%\right.$ agar), while $T$. rubrum was cultured at $26{ }^{\circ} \mathrm{C}$ in Sabouraud Dextrose broth and Sabouraud Dextrose agar $(0.75 \%$ agar). The three powdered extracts, DRVE, FRVE, and FFRVE, were dissolved in water to obtain concentrations of 100 and $1000 \mathrm{mg} / \mathrm{mL}$ for each extract. Fifty microliters of each extract was injected into a sterile disk of 6-mm diameter (Toyo Roshi Kaisha Ltd., Tokyo, Japan), and the solvent was allowed to dry off in an aseptic hood. Accordingly, disks were loaded with 5 and $50 \mathrm{mg}$ of each crude extract. Standard disks containing distilled water served as negative controls for the antimicrobial test.

After $24 \mathrm{~h}, P$. acnes or $T$. rubrum cultures were adjusted to $1 \times 10^{8} \mathrm{CFU} / \mathrm{mL}$ using $0.5 \mathrm{McF}$ arland standards and inoculated into Mueller Hinton agar and Sabouraud
Dextrose agar, respectively. Next, disks containing the extracts were placed on a plate and incubated at 37 and $26{ }^{\circ} \mathrm{C}$, respectively. $P$. acnes and T. rubrum plates were incubated for 2 and 5 days, respectively. The antimicrobial activities of the extracts were then determined by measuring the diameter of the clear zones around the disks in millimeters. This measurement was carried out in triplicate.

\section{Cell culture}

Human hepatocellular carcinoma (HepG2, ATCC HB8065) and murine normal hepatocyte (AML12, ATCC CRL-2254) cell lines were maintained in Dulbecco's modified Eagle's medium (DMEM) containing 10\% fetal bovine serum (FBS, Welgene, Daegu, Korea) with $1 \%$ antibiotics (Welgene). Human prostate cancer (PC3, ATCC CRL-1435), breast cancer (MDA-MB-231, ATCC HTB-26), and colon cancer (HCT116, ATCC CCL-247) cell lines were maintained in RPMI 1640 containing $10 \%$ FBS (Welgene) with $1 \%$ antibiotics (Welgene).

\section{Cell viability assay}

HepG2, PC3, MDA-MB-231, HCT116, and AML12 cells were incubated in 96 -well plates $\left(5 \times 10^{4}\right.$ cells/well $)$ and treated with various doses of DRVE, FRVE, and FFRVE $(0,50,100,200$, and $400 \mu \mathrm{g} / \mathrm{mL})$ for $24 \mathrm{~h}$. Next, $50 \mu \mathrm{L}$ of 3-(4-5-dimethylthiazol-2-yl)-2,5-diphenyl tetrazolium bromide (MTT, $1 \mathrm{mg} / \mathrm{mL}$, Sigma-Aldrich, St. Louis, MO, USA) was added. After $2 \mathrm{~h}$, formazan crystals in viable cells were dissolved in dimethyl sulfoxide (DMSO), and cell viability was calculated by measuring the optical density (OD) at $570 \mathrm{~nm}$ using a microplate reader (Molecular Devices Co, Sunnyvale, CA, USA).

\section{High-performance liquid chromatography}

Urushiol in the three extracts was analyzed by high-performance liquid chromatography (HPLC, Agilent Technologies, Santa Clara, CA, USA) using Hichrome HPLC columns $(5 \mu \mathrm{m}, 250 \mathrm{~mm} \times 4.6 \mathrm{~mm}$, Hichrome, Ltd., Theale, UK). A flow rate of $0.3 \mathrm{~mL} / \mathrm{min}$ and an injection volume of $10 \mu \mathrm{L}$ were used. The solvent used was $100 \%$ methanol, and the detection wavelength was set to $254 \mathrm{~nm}$.

Moreover, the polyphenols gallic acid, fustin, fisetin, quercetin, butein, and sulfuretin were analyzed in the three RVS extracts via HPLC. The mobile phases were composed of $0.1 \%$ formic acid in water (solvent $\mathrm{A}$ ) and $100 \%$ methanol (solvent B), delivered at a flow rate of $0.7 \mathrm{~mL} / \mathrm{min}$. The following gradient conditions were used: $0-17 \mathrm{~min}, 100 \% \mathrm{~B} ; 17-20 \mathrm{~min}, 100 \% \mathrm{~B} ; 20$ $23 \mathrm{~min}, 0 \% \mathrm{~B}$; and $23-30 \mathrm{~min}, 0 \% \mathrm{~B}$. The detection wavelength was set to $254 \mathrm{~nm}$, and an injection volume of $10 \mu \mathrm{L}$ was used. 


\section{Oil red $O$ staining}

HepG2 cells were incubated in a 6 -well plate $\left(5 \times 10^{5}\right.$ cells/well) for $24 \mathrm{~h}$. The cells were then treated with DRVE, FRVE, and FFRVE $(400 \mu \mathrm{g} / \mathrm{mL})$, and stimulated with OA $(200 \mu \mathrm{M})$ for a further $24 \mathrm{~h}$. After incubation, the supernatants were discarded and cells were washed with phosphate-buffered saline (PBS). Cell fixation was done by treating with $4 \%$ formalin for $10 \mathrm{~min}$, followed by washing with PBS and staining with Oil Red O (ORO). ORO stain was extracted using isopropanol and the OD was measured at $510 \mathrm{~nm}$ using a microplate reader (Molecular Devices Co).

\section{Western blot analysis}

HepG2 cells were incubated in a 6 -well plate $\left(5 \times 10^{5}\right.$ cells/well) for $24 \mathrm{~h}$. The cells were then treated with DRVE, FRVE, and FFRVE $(400 \mu \mathrm{g} / \mathrm{mL})$, and stimulated with OA $(200 \mu \mathrm{M})$ for a further $24 \mathrm{~h}$ at $37{ }^{\circ} \mathrm{C}$. Next, cells were harvested and lysed with radioimmunoprecipitation assay (RIPA) buffer (50 mM Tris- $\mathrm{HCl}$ (pH 7.4), $150 \mathrm{mM}$ $\mathrm{NaCl}, 1 \% \mathrm{NP}-40,0.25 \%$ sodium deoxycholate, $1 \mathrm{M}$ EDTA, $1 \mathrm{mM} \mathrm{Na}_{3} \mathrm{VO}_{4}, 1 \mathrm{mM} \mathrm{NaF}$, and protease-inhibitor cocktail). Protein samples were quantitated using a Bio-Rad DC protein assay kit II (Bio-Rad, Hercules, CA, USA), resolved via sodium dodecyl sulfate-polyacrylamide gel electrophoresis (SDS-PAGE) on $8 \%$ polyacrylamide gel, and electrotransferred onto a BioTrace NT transfer membrane (Pall, Gelman Laboratory, Port Washington, NY, USA). After electrotransfer, membranes were blocked with 5\% skimmed milk (BD, NJ, USA) and probed with primary antibodies for sterol regulatory element-binding protein 1 (SREBP-1, Santa Cruz Biotechnology, Santa Cruz, CA, USA), peroxisome proliferator-activated receptor alpha (PPAR- $\alpha$, Santa Cruz Biotechnology), AMP-activated protein kinase (AMPK, Cell Signaling, Denvers, MA, USA), phosphorylated AMPK (p-AMPK, Cell Signaling), and $\beta$-actin (Sigma-Aldrich) overnight, and exposed to horseradish peroxidase-conjugated secondary anti-mouse or anti-rabbit antibodies. Protein expression levels were detected using an EZ-Western Lumi Pico kit (DOGEN, Seoul, Korea).

\section{Measurement of cellular triglyceride levels}

HepG2 cells were incubated in a 6 -well plate $\left(5 \times 10^{5}\right.$ cells/well) for $24 \mathrm{~h}$. The cells were then treated with DRVE, FRVE, and FFRVE $(400 \mu \mathrm{g} / \mathrm{mL})$, and stimulated with OA $(200 \mu \mathrm{M})$ for a further $24 \mathrm{~h}$. To measure cellular triglyceride (TG) levels, a chloroform-methanol extraction method was applied with some modifications, as described in a previous protocol $[12,13]$. Cells were collected and mixed with $1 \mathrm{~mL}$ of a 2:1 chloroform:methanol mixture at room temperature for $20 \mathrm{~min}$. After centrifugation at $500 \times \mathrm{g}$ for $10 \mathrm{~min}$, the lower layers were collected and dried overnight at $4^{\circ}$
$\mathrm{C}$, and TG levels were measured using a TG assay kit (Asan Pharm, Hwaseong-si, Korea). The OD was measured at $550 \mathrm{~nm}$ using a microplate reader (Molecular Devices Co).

\section{Determination of the antioxidant capacity of the three extracts}

The 1,1-diphenyl-2-picrylhydrazyl (DPPH) radical scavenging activity was measured by the Blois method [14]. The extracts were mixed with DPPH and stabilized at room temperature for $30 \mathrm{~min}$. The OD was then measured at $515 \mathrm{~nm}$ using a microplate reader (Molecular Devices Co).

\section{Statistical analysis}

Results are presented as means \pm standard deviation of triplicates. Data were analyzed via Student's $t$-test. Differences among groups were considered statistically significant if $p<0.05$.

\section{Results \\ Confirmation of DRVE, FRVE, and FFRVE detoxification}

To confirm detoxification of the three RVS extracts, the presence of the allergen urushiol was investigated by HPLC. Urushiol (Fig. 1a) was not detected in any of the three extracts (Fig. 1b, c, and d), thus confirming successful detoxification. The urushiol peak in the standard appeared at $13.62 \mathrm{~min}$.

\section{DRVE demonstrated the strongest antioxidant activity among the three extracts}

The antioxidant activities of DRVE, FRVE, and FFRVE were evaluated via a DPPH radical scavenging assay. Quercetin, tannic acid, and ascorbic acid were used as standards to analyze DPPH scavenging capacity.

The half-maximal effective concentration $\left(\mathrm{EC}_{50}\right)$ of any antioxidant compound is inversely related to its antioxidant activity, as it is the concentration of the antioxidant needed to decrease the radical concentration by $50 \%$. Thus, lower $\mathrm{EC}_{50}$ values indicate higher antioxidant activities. DRVE and FRVE exhibited $\mathrm{EC}_{50}$ values of 48.7 and $111.3 \mu \mathrm{g} / \mathrm{mL}$, respectively, while the DPPH radical scavenging activity of FFRVE was $28.6 \%$ at its high concentration $(200 \mu \mathrm{g} / \mathrm{mL}$, Table 1$)$. The radical scavenging activity of DRVE was stronger than that of the two other extracts (Table 1).

\section{FRVE demonstrated the strongest antimicrobial activity among the three extracts}

The antimicrobial activities of the three RVS extracts were investigated against two different microbes that cause skin diseases ( $P$. acne, an acne-causing bacterium, and T. rubrum, a fungus that affects hands and nails and causes athlete's foot) using an agar diffusion 


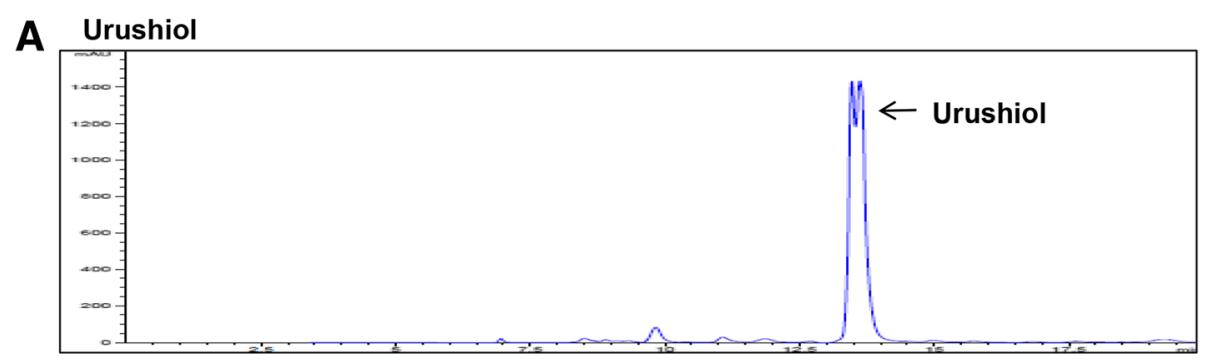

B DRVE

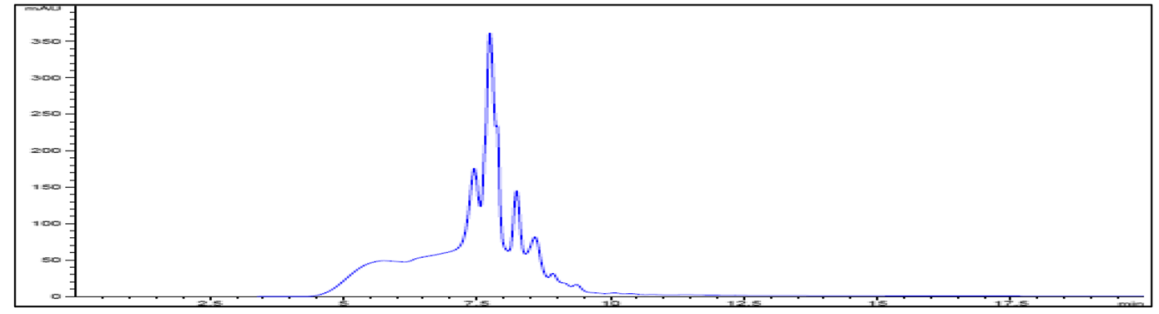

C FRVE

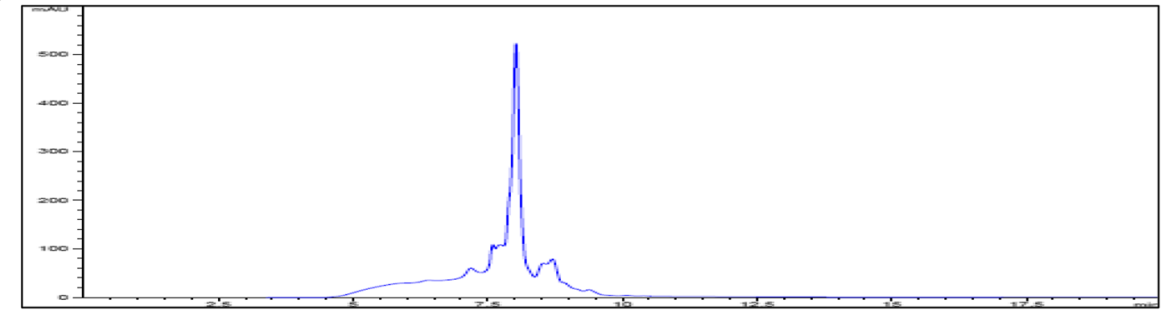

D FFRVE

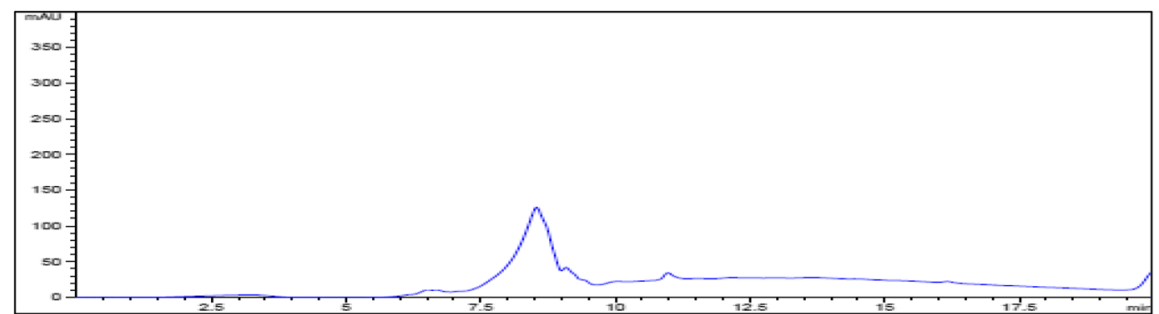

Fig. 1 Determination of allergen-free Rhus verniciflua Stokes extracts (DRVE, FRVE, FFRVE) by HPLC analysis. HPLC analysis identified urushiol as an allergen of Rhus verinciflua Stokes. a Urushiol standard b DRVE c FRVE d FFRVE

method at doses of 5 and $50 \mathrm{mg} /$ disk. Only the $50 \mathrm{mg} /$ disk dose of FRVE demonstrated a strong antimicrobial activity against $P$. acnes (Fig. 2a) and T. rubrum

Table 1 Antioxidant capacity of the DRVE, FRVE, and FFRVE by DPPH scavenging assay

\begin{tabular}{ll}
\hline Sample & DPPH, EC50 $(\mu \mathrm{g} / \mathrm{ml})$ \\
\hline DRVE & $48.7 \pm 2.1$ \\
FRVE & $111.3 \pm 1.3$ \\
FFRVE & $>200(28.6 \%)$ \\
Quercetin & $7.7 \pm 0.1$ \\
Tannic acid & $8.4 \pm 0.5$ \\
Ascorbic acid & $11.8 \pm 1.1$
\end{tabular}

Each value represents mean $\pm \mathrm{SD}(n=3)$ cultures (Fig. 2b), producing 42- and 25-mm inhibition zones, respectively.

The anticancer effects of DRVE, FRVE, and FFRVE DRVE and FRVE inhibit cancer cell viability without affecting normal cells

We tested the effects of DRVE, FRVE, and FFRVE on the viability of four human cancer cell lines (HepG2, PC3, MDA-MB-231, and HCT116) and of normal cells (AML-12). The concentrations of FRVE and DRVE tested were $0,50,100,200$, and $400 \mu \mathrm{g} / \mathrm{mL}$.

As shown in Fig. 3a, the viability of HepG2 cells was reduced to 29,22 , and $12 \%$ when incubated with $400 \mu \mathrm{g} / \mathrm{mL}$ DRVE, FRVE, and FFRVE, respectively, for $24 \mathrm{~h}$ (Fig. 3a). Similarly, treatment with $400 \mu \mathrm{g} / \mathrm{mL}$ DRVE, FRVE, and FFRVE reduced prostate cancer cell 


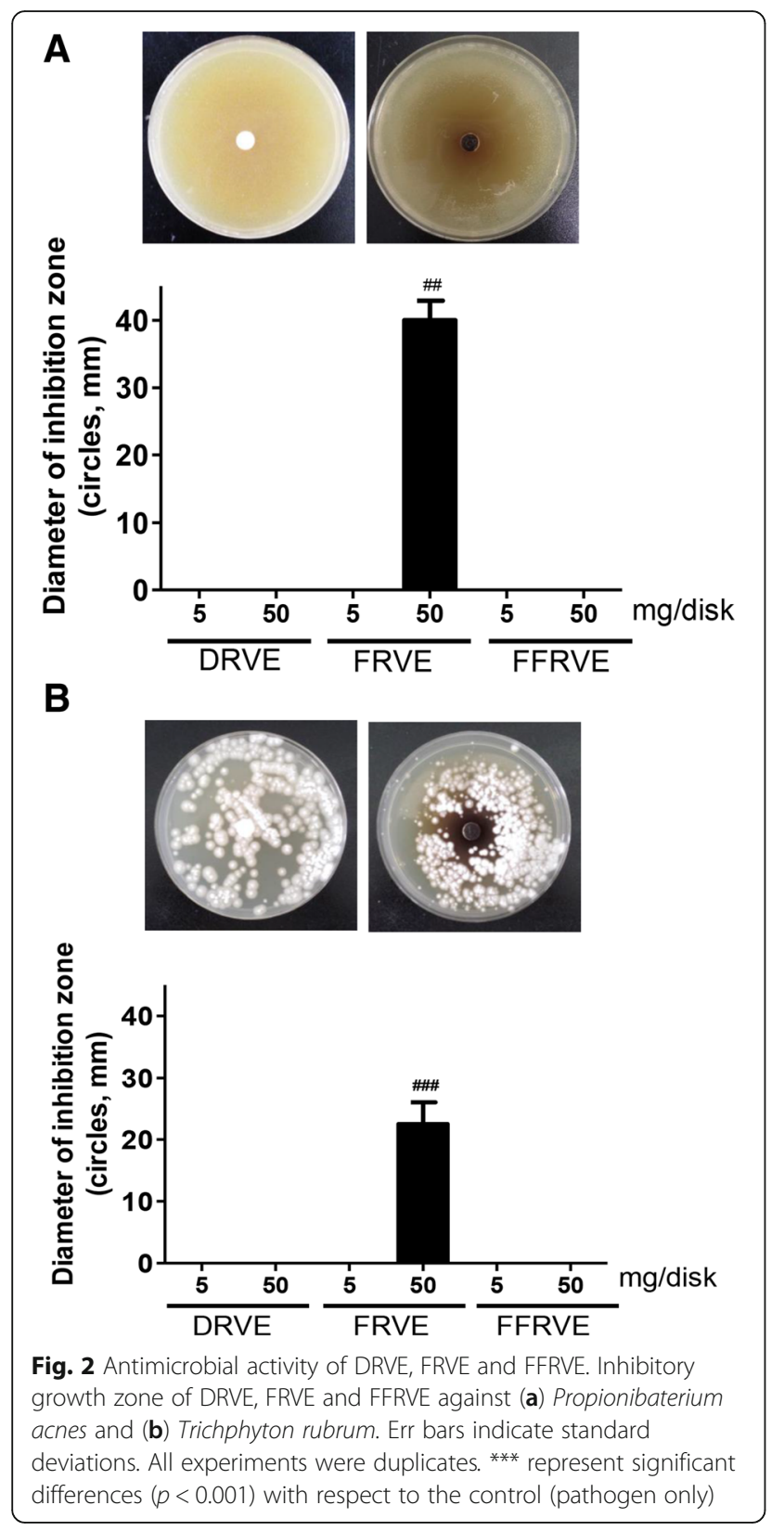

viability by 39,37 , and $21 \%$, respectively (Fig. 3 b). Of all the cancer cell lines studied, the breast cancer cell line MDA-MB-231 showed the lowest viability (11\%) after treatment with $400 \mu \mathrm{g} / \mathrm{mL}$ DRVE (Fig. 3c). With regard to colorectal cancer cells, DRVE and FRVE treatments showed similar effects on the viabilities of HCT116 cells (28 and 31\%, respectively), whereas viability was approximately twice greater after FFRVE treatment (67\%, Fig. 3d). Overall, DRVE exhibited the strongest cytotoxic effect against cancer cells.

In contrast, DRVE and FRVE did not affect the viability of normal cells (AML-12), even at the highest concentration tested (Fig. 3e), while $400 \mu \mathrm{g} / \mathrm{mL}$
FFRVE inhibited the growth of AML-12 cells by $8 \%$ (Fig. 3e).

The three RVS extracts suppressed hepatic lipogenesis in an in vitro model of non-alcoholic fatty liver

We previously reported that FRVE could inhibit hepatic lipogenesis in OA-treated HepG2 cells. In order to investigate whether DRVE and FFRVE could alleviate OA-induced cellular steatosis, OA-treated HepG2 cells were stained with ORO. As shown in Fig. 4a, DRVE decreased the number and size of lipid droplets in OA-treated HepG2 cells. DRVE, FRVE, and FFRVE treatments decreased the fat deposits by 22, 34, and $11 \%$, respectively, compared with that in OA-treated HepG2 cells not treated with extracts (Fig. 4a).

To evaluate the regulatory effect of the extracts on lipogenesis and fatty acid oxidation, the levels of SREBP1 , PPAR- $\alpha$, and AMPK in HepG2 cells were determined via western blotting. Our results showed that the three RVS extracts downregulated OA-induced elevation of SREBP-1 levels in HepG2 cells. FRVE and DRVE increased the expression of PPAR- $\alpha$ and p-AMPK, while FFRVE increased PPAR- $\alpha$ but not $\mathrm{p}$-AMPK expression levels (Fig. 4b). In order to assess OA-induced TG accumulation, and the effects of the extracts on it, cellular TG levels were measured. OA-treated cells exhibited elevated TG levels compared to non-treated cells. However, treatment with DRVE, FRVE, and FFRVE lowered TG levels to 28, 35, and 17\%, respectively (Fig. 4c).

Although the three extracts demonstrated lipid-lowering effects in the in vitro model of non-alcoholic fatty liver, DRVE and FRVE were more effective than FFRVE at the same concentration.

\section{Comparison of the polyphenol content in the three RVS extracts}

Gallic acid, fustin, fisetin, quercetin, butein, and sulfuretin are the main active constituents of $R$. verniciflua. We compared the contents of four phenolic compounds (gallic acid, fustin, fisetin, and quercetin) as marker compounds in DRVE, FRVE, and FFRVE via HPLC. The retention times of gallic acid, fisetin, fustin, and quercetin were $10.045,13.033,16.751$, and $17.631 \mathrm{~min}$, respectively (Fig. 5a).

These polyphenols were detected in DRVE, FRVE, and FFRVE eluents (Fig. 5b, c, and d, respectively). Gallic acid levels in DRVE, FRVE, and FFRVE were 170, 33, and $4 \mathrm{mg} / \mathrm{g}$, respectively, and gallic acid content in DRVE was therefore 42.5 times greater than that in FFRVE (Table 2). Fustin levels in DRVE, FRVE, and FFRVE were $34.5,129$, and $10.7 \mathrm{mg} / \mathrm{g}$, respectively, and fustin content in FRVE was therefore 12 times higher than that in FFRVE (Table 2). Fisetin levels in DRVE, FRVE, and FFRVE were 15, 59, and $1 \mathrm{mg} / \mathrm{g}$, respectively, 


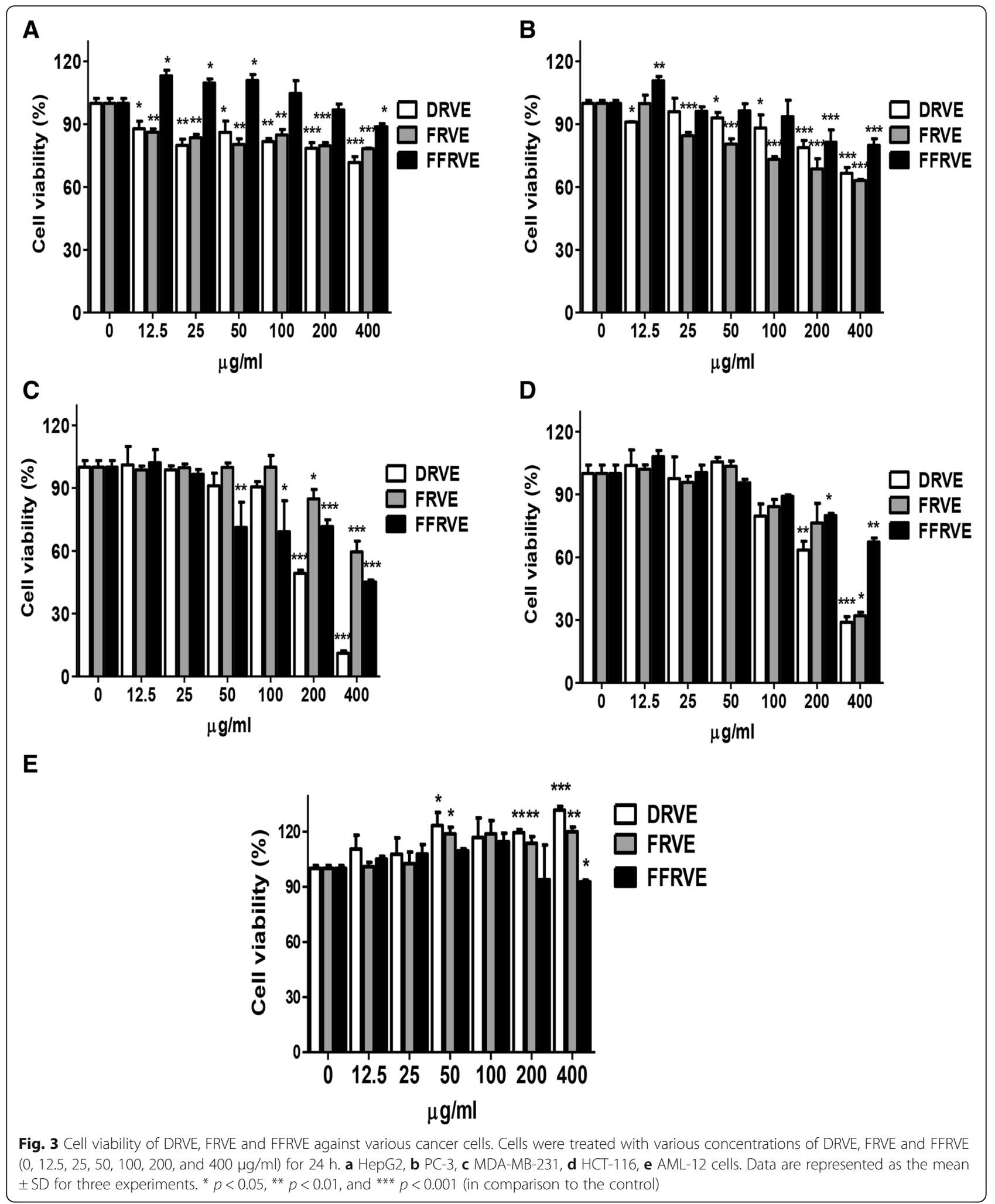

and fisetin content was therefore 59 times greater in FRVE than in FFRVE. Finally, quercetin levels in DRVE, FRVE, and FFRVE were $1.5,4$, and $19 \mathrm{mg} / \mathrm{g}$, respectively (Table 2).
The four marker compounds of DRVE, FRVE, and FFRVE possess antioxidant activities

According to the DPPH scavenging assay, the antioxidant activities of the evaluated compounds could be 


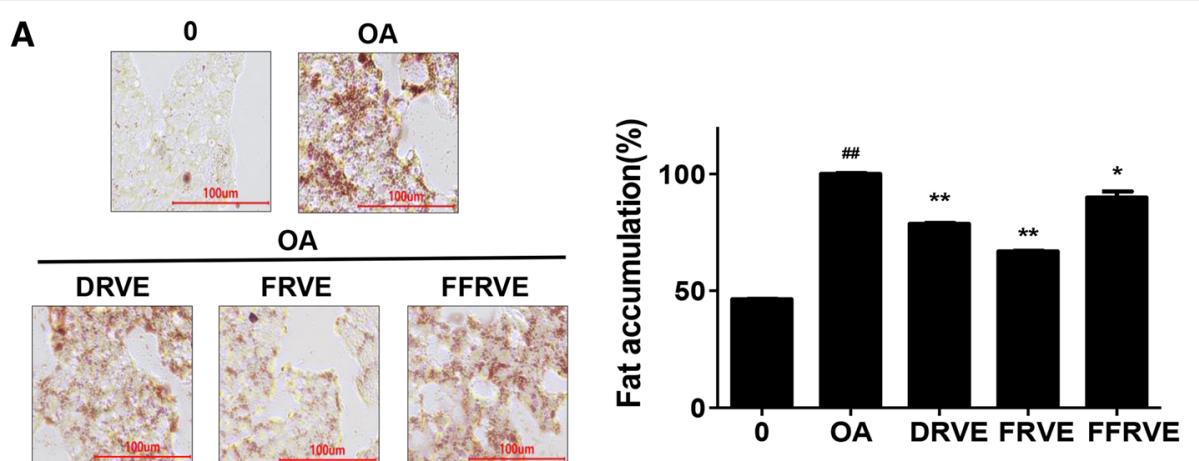

B
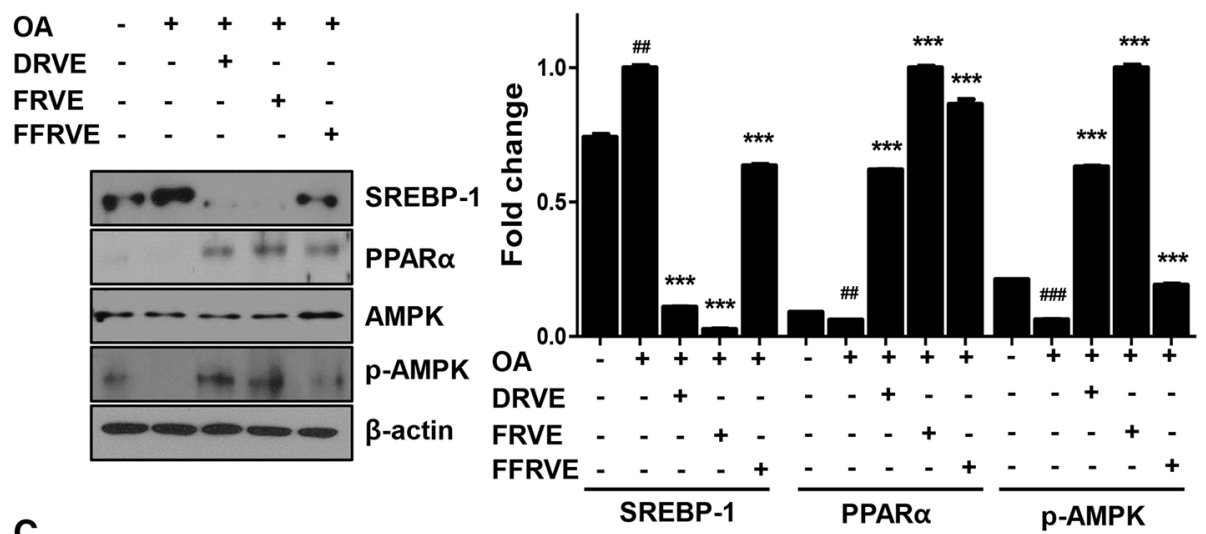

C

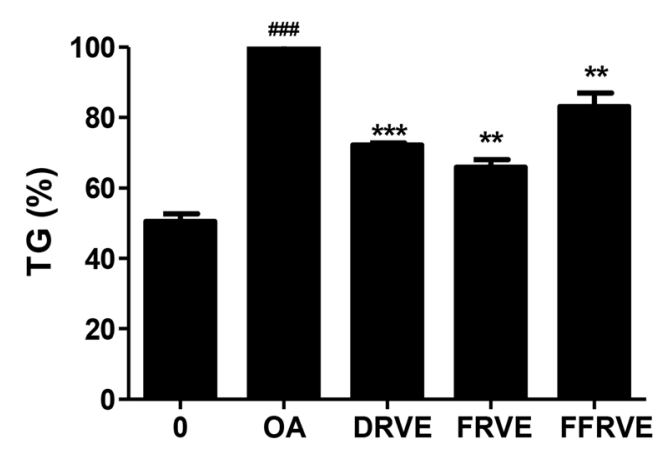

Fig. 4 Effect of DRVE, FRVE, FFRVE on cellular lipid accumulation and lipogenesis in OA-induced HepG2 cells. Cells were co-treated with OA and $400 \mathrm{\mu g} / \mathrm{ml}$ of DRVE, FRVE, FFRVE for $24 \mathrm{~h}$. a Cells were stained with ORO as described in the materials and methods and then quantitatively analyzed. ORO staining image (magnification 400X), (\#\#) $p<0.01$ (in comparison to the non-OA treated control), * and ** represent significant differences ( $p<0.05$ and $p<0.01$, respectively) with respect to the OA-treated control. $\mathbf{b}$ Determination of the expression of AMPK, SREBP-1 and PPARa by western blotting analysis. Quantitative protein levels were shown. (\#\#) $p<0.01$, and (\#\#\#) $p<0.001$ (in comparison to the non-OAtreated control). ( $* *) p<0.01$ and $(* * *) p<0.001$ (in comparison to the OA-treated control). c Total intracellular TG was analyzed by an enzymatic colorimetric method. Data are represented as the mean \pm SD of three experiments. (\#\#\#) $p<0.001$ (in comparison to the non-OA treated control). $(* *) p<0.01$ and $(* * *) p<0.001$ (in comparison to the OA-treated control)

ordered as follows: fisetin > gallic acid > ascorbic acid > quercetin $>$ fustin (Table 3).

Fisetin showed the strongest radical scavenging activity and the lowest $\mathrm{EC}_{50}$ value $(1 \pm 0.01 \mu \mathrm{g} / \mathrm{mL})$, whereas fustin exhibited the lowest DPPH scavenging activity and the highest $\mathrm{EC}_{50}$ value $(15.1 \pm 0.04 \mu \mathrm{g} / \mathrm{mL}$, Table 3$)$.

The relative antioxidant capacities of DRVE and FRVE (Table 1) were similar to those of gallic acid and fustin, respectively.

\section{Discussion}

Despite the various benefits of RVS, it is known to cause allergy, which may be due to the presence of urushiol. Nevertheless, due to its numerous biological activities, RVS is of high importance in the development of functional food and medicine. Therefore, various detoxification methods have been developed for the removal of allergens from RVS. In Korea, the Ministry of Food and Drug Safety permitted the use of 
A
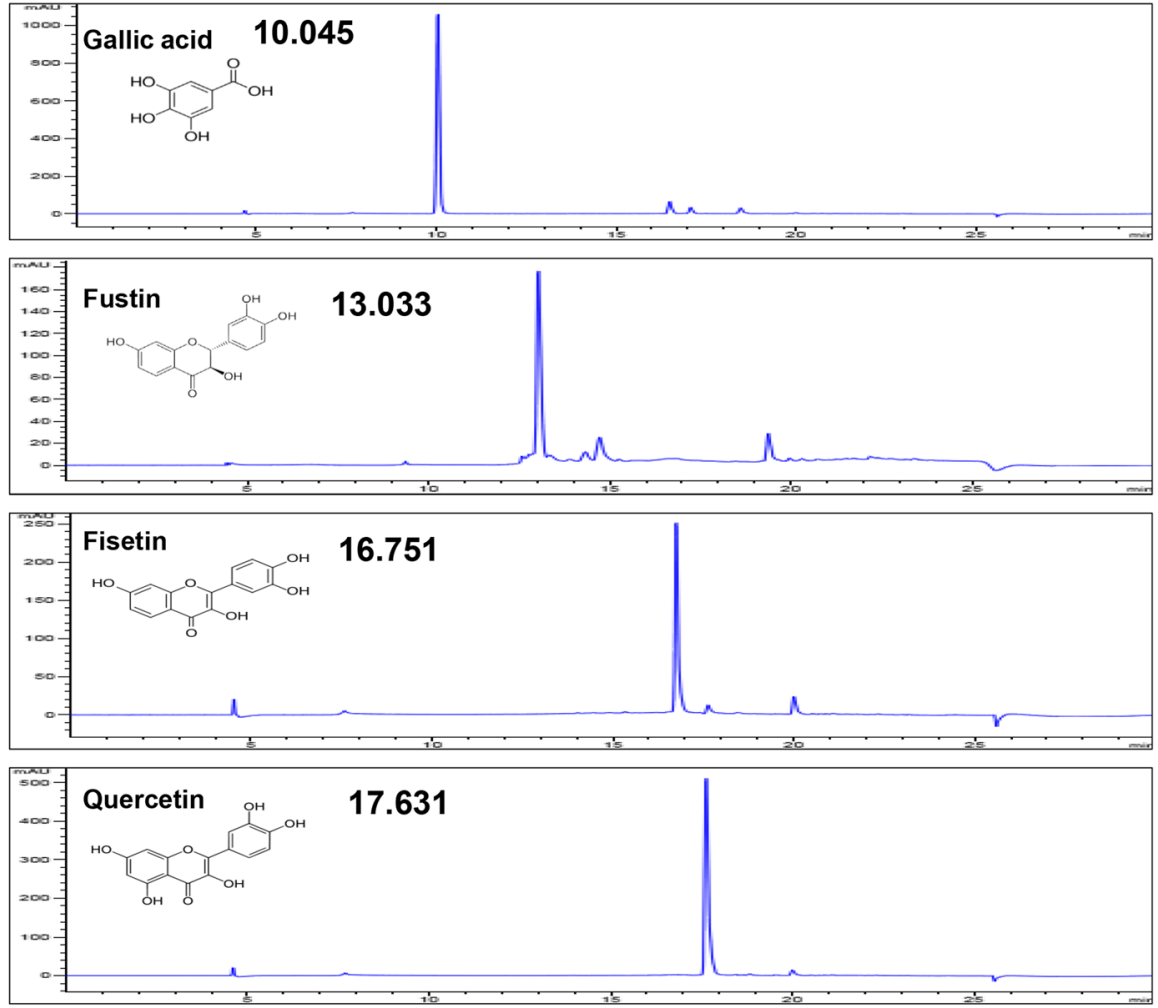

B

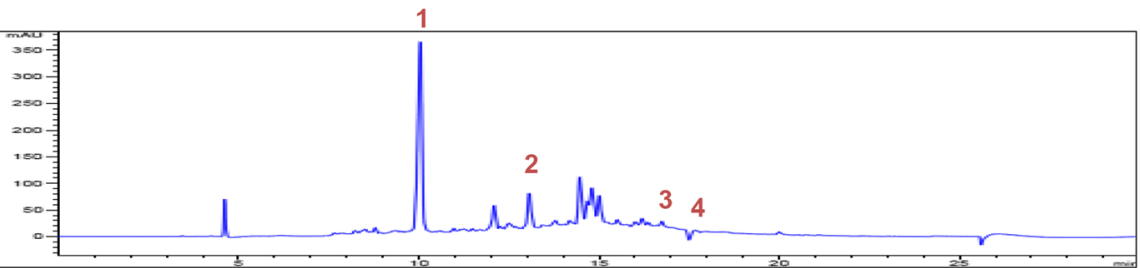

C

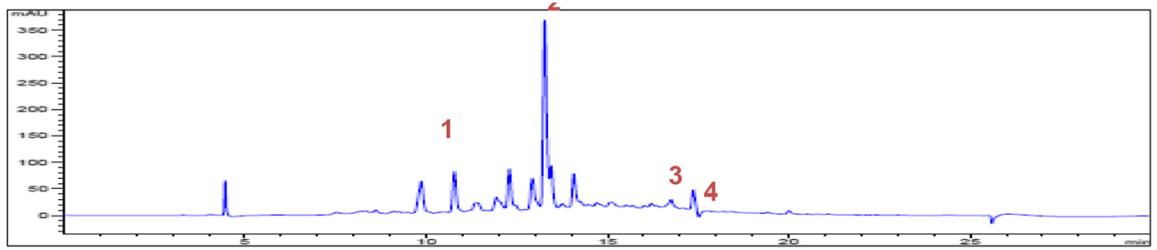

D

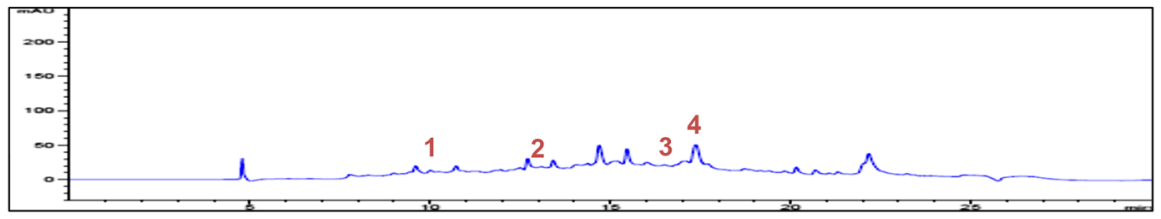

Fig. 5 HPLC chromatograms of the DRVE, FRVE, and FFRVE. Peaks of four main components (a) are gallic acid (1), fustin (2), fisetin (3), quercetin (4) and DRVE (b), FRVE (c), FFRVE (d). Mobile phase: $0.1 \%(v / v)$ Formic acid in water (solvent A) and 100\% (v/v) Methanol (solvent B) at a flow rate of $0.7 \mathrm{ml} / \mathrm{min}$, with gradient as follows: 0-17 min, 100\%B; 17-20 min, 100\%B; 20-23 min, 0\%B; 23-30 min, 0\%B. and detected at $254 \mathrm{~nm}$

detoxified RVS extracts in 2012. Since then, the types of functional foods containing detoxified RVS extracts have been steadily increasing on a yearly basis. Detoxification methods include the removal of allergens from
RVS using solvents [15], electron beam radiation [16], high temperature [17], and microorganisms [9, 13]. However, RVS extracts detoxified by solvents and irradiation are not suitable for use in food. 
Table 2 Contents of Phenolic acids in DRVE, FRVE, and FFRVE

\begin{tabular}{lllll}
\hline Sample & \multicolumn{4}{l}{ Compound $(\mathrm{mg} / \mathrm{g})$} \\
\hline DRVE & Gallic acid & Fustin & Fisetin & Quercetin \\
FRVE & 170 & 34.5 & 15 & 1.5 \\
FFRVE & 33 & 129 & 59 & 4 \\
Sample & 4.3 & 10.7 & 19 & 7 \\
\hline
\end{tabular}

We have been studying the biological activities of RVS along with many other researchers, and to date, several bioactivities have been reported. However, studies on detoxified RVS extracts are still inadequate.

To the best of our knowledge, there are no studies comparing RVS extracts prepared by different detoxification methods. Thus, the present study was conducted to compare the bioactive constituents and biological activities of three RVS extracts prepared by different detoxification methods. The investigated extracts (DRVE, FRVE, and FFRVE) are commercially available as allergen-free functional food in Korea.

The antioxidant activities of the three extracts were assessed via DPPH scavenging assay. Among the three extracts, DRVE was found to possess a superior antioxidant activity. Regarding antimicrobial activity, FRVE was the most effective, and inhibited the growth of both $P$. acnes and $T$. rubrum. The three extracts successfully suppressed hepatic lipogenesis in an in vitro model of non-alcoholic fatty liver. However, DRVE and FRVE were more effective than FFRVE at the same concentration. These results suggest that the different detoxification methods may induce alterations in the major components of RVS, leading to differences in their activities. Therefore, we analyzed the polyphenolic constituents of RVS in the three extracts.

Polyphenols are bioactive compounds present at high concentrations in various plants [18]. Many studies have reported that phenolics such as a fustin, fisetin, gallic acid, and quercetin are highly abundant compounds in R. verniciflua $[3,19,20]$.

According to a previous report, RVS extracts detoxified via heating methods show high gallic acid contents [17]. This is consistent with our finding that DRVE, an allergen-free RVS extract detoxified by heating to a high

Table 3 Antioxidant effect of four main compounds by DPPH scavenging assay

\begin{tabular}{ll}
\hline Sample & DPPH, EC50 $(\mu \mathrm{g} / \mathrm{ml})$ \\
\hline Ascorbic acid & $8.1 \pm 0.6$ \\
Gallic acid & $1.8 \pm 0.1$ \\
Fustin & $15.1 \pm 0.0$ \\
Fisetin & $1 \pm 0.0$ \\
Quercetin & $7.9 \pm 0.2$
\end{tabular}

Each value represents mean $\pm \operatorname{SD}(n=3)$ temperature, possesses the highest gallic acid content. Moreover, another study reported that an allergen-free RVS extract detoxified by heating (by roasting in an iron pot at $240{ }^{\circ} \mathrm{C}$ for $50 \mathrm{~min}$ and extracting with water) contains higher amounts of fustin $(130 \mathrm{mg} / \mathrm{g})$ than fisetin (20 mg/g) [21]. Similar to these data, our results demonstrated that DRVE contained three times more fustin than fisetin. However, FFRVE, the RVS extract detoxified by fermentation with $F$. fraxinea mushroom, was found to contain more fisetin than fustin. This result is in agreement with that reported in a previous study [7]. Finally, FRVE, the RVS extract detoxified by fermentation with the yeast $S$. carlsbergensis, contained higher levels of fustin than fisetin or gallic acid, unlike DRVE or FFRVE. As shown in Tables 2 and 3, DRVE was found to contain the highest amount of gallic acid among the three extracts, and gallic acid was found to be the second most effective antioxidant after fisetin. Therefore, gallic acid was considered to be a marker compound reflecting the antioxidant effect of DRVE, while fustin was considered to be a marker compound reflecting the bioactivity of FRVE. However, the contents of all marker compounds, including fisetin, were low. Therefore, FFRVE seems to have a low biological activity when compared to DRVE or FRVE.

\section{Conclusions}

The present study reports a comparison between the components and biological activities of three different kinds of commercially available allergen-free RVS extracts in Korea. Our findings suggest that the components may vary according to the detoxification method used. Accordingly, by altering the detoxification method, it is possible to maximize the concentrations of components that exert specific effects for application in the health food or cosmetic product industry.

\section{Abbreviations}

AMPK: AMP-activated protein kinase; D.W: Distilled Water; DMSO: Dimethyl sulfoxide; DPPH: 1,1-diphenyl-2-picrylyhdrazyl; DRVE: Dried Rhus verniciflua STOKES extract; $E_{50}$ : Half maximal effective concentration; FBS: Fetal Bovine Serum; FFRVE: Fermented with Fomitella fraxinea Rhus verniciflua STOKES extract; FRVE: Fermented Rhus verniciflua STOKES extract; HRP: Horseradish peroxidase; MTT: 3-(4-5-dimethylthiazol-2-yl)-2,5-diphenyle tetrazolium bromide; O.D: Optical density; OA: Oleic acid; PBS: Phosphate-buffered saline; milk; PPARPa: Peroxisome proliferator-activated receptor alpha; RVS: Rhus verniciflua STOKES; SD: Standard deviation; SREBP-1: Sterol regulatory element-binding protein 1; TG: Triglyceride

\section{Funding}

This work was supported by grants (No. 2013R1A1A1008431) from the National Research Foundation of Korea (NRF) (MSIP, Ministry of Science, ICP and Future Planning) and "Cooperative Research Program for Agriculture Science and Technology Development (Project No. PJ01313501)" Rural Development Administration, Republic of Korea.

Availability of data and materials

All data generated or analysed for this study are included in this publish article. (However, raw data are available from the corresponding author on reasonable request. 


\section{Authors' contributions}

HJL conceived and designed the experiments; SOL, SJL, and HJL performed the experiments; EOL analyzed the data; and JSK contributed reagents/ analysis tools. All authors contributed in validating, writing and approving the final version of the manuscript.

\section{Ethics approval and consent to participate}

N/A

\section{Consent for publication}

Not applicable.

\section{Competing interests}

The authors declare that they have no competing interests.

\section{Publisher's Note}

Springer Nature remains neutral with regard to jurisdictional claims in published maps and institutional affiliations.

\section{Author details}

'Department of Science in Korean Medicine, Graduate School, Kyung Hee University, Hoegi-dong, Dongdaemun-gu, 130-701 Seoul, Republic of Korea. ${ }^{2}$ Department of Cancer Preventive Material Development, Graduate School, Kyung Hee University, Hoegi-dong, Dongdaemun-gu, 130-701 Seoul, Republic of Korea. ${ }^{3}$ College of Korean Medicine, Kyung Hee university, 1 Hoegi-dong, Dondaemun-gu, 130-701 Seoul, Republic of Korea. ${ }^{4}$ Major of Plant Resources and Environment, College of Applied Life Sciences, 102 Jeju National University, Jeju-si, Jeju-do 690-756, Korea.

\section{Received: 20 February 2018 Accepted: 22 August 2018}

\section{Published online: 30 August 2018}

\section{References}

1. Yoo H, Roh J. Compendium of Prescriptions From the Countryside (Hyangyakjipseongbang), vol. 1433. Seoul: Hangrimchulpansa; 1977.

2. Kim J-S, Kwon Y-S, Chun W-J, Kim T-Y, Sun J, Yu C-Y, Kim M-J. Rhus verniciflua Stokes flavonoid extracts have anti-oxidant, anti-microbial and aglucosidase inhibitory effect. Food Chem. 2010;120:539-43.

3. Kim KH, Moon E, Choi SU, Kim SY, Lee KR. Polyphenols from the bark of Rhus verniciflua and their biological evaluation on antitumor and antiinflammatory activities. Phytochemistry. 2013;92:113-21.

4. Kim M-O, Kim J-S, Sa Y-J, Jeong H-J, Chun W-J, Kwon Y-S, Kim T-Y, Choi H-S, Yu C-Y, Kim M-J. Screening of extraction solvent condition of fermented Rhus verniciflua stem bark by antioxidant activities. Korean J Med Crop Sci. 2010;18:217-23.

5. Kim M-J, Choi W-C, Barshinikov A, Kobayashi A. Anticancer and antioxidant activity of allergen-removed extract in Rhus verniciflua Stokes. Korean J Med Crop Sci. 2002:10:288-93.

6. M-o K, Yangb J, Kwonc YS, Kima MJ. Antioxidant and anticancer effects of fermented Rhus verniciflua stem bark extracts in HCT-116 cells. SCIENCEASIA. 2015:41:322-8.

7. Choi H-S, Yeo S-H, Jeong S-T, Choi J-H, Park H-S, Kim M-K. Preparation and characterization of urushiol free fermented Rhus verniciflua stem bark (FRVSB) extracts. Korean J Food Sci Technol. 2012;44:173-8.

8. Kobayashi S, Ikeda R, Oyabu H, Tanaka H, Uyama H. Artificial Urushi: design, synthesis, and enzymatic curing of new urushiol analogues. Chem Lett. 2000;29:1214-5.

9. Choi H-S, Kim M-K, Park H-S, Yun S-E, Mun S-P, Kim J-S, Sapkota K, Kim S, Kim T-Y, Kim S-J. Biological detoxification of lacquer tree (Rhus verniciflua Stokes) stem bark by mushroom species. Food Sci Biotechnol. 2007;16:935-42.

10. Bauer A, Kirby W, Sherris JC, Turck M. Antibiotic susceptibility testing by a standardized single disk method. Am J Clin Pathol. 1966;45:493.

11. Rios J, Recio M, Villar A. Screening methods for natural products with antimicrobial activity: a review of the literature. J Ethnopharmacol. 1988;23: 127-49.

12. Folch J, Lees $\mathrm{M}$, Sloane Stanley $\mathrm{GH}$. A simple method for the isolation and purification of total lipides from animal tissues. J Biol Chem. 1957;226:497-509.

13. Lee MS, Kim JS, Cho SM, Lee SO, Kim SH, Lee HJ. Fermented Rhus verniciflua Stokes extract exerts an Antihepatic Lipogenic effect in oleic-acid-induced HepG2 cells via Upregulation of AMP-activated protein kinase. J Agric Food Chem. 2015;63:7270-6.
14. Blois MS. Antioxidant determinations by the use of a stable free radical. Nature. 1958;181:1199-200.

15. Choi WC, Park SJ, Kwon SP. Process for preparation of rhus verniciflua extracts having excellent anti-cancer activity and anti-cancer pharmaceutica composition containing the same. U.S. patent US7618661B2, November 17, 2009

16. Kim B. The effect of irradiation and peroxyacetic acid treatment on the reduction of urushiol of Rhus verniciflua Stokes and the physiological activities. Gwangju: MS thesis, Chonbuk National University; 2010.

17. Liu C-S, Nam T-G, Han M-W, Ahn S-M, Choi HS, Kim TY, Chun OK, Koo SI, Kim D-O. Protective effect of detoxified Rhus verniciflua stokes on human keratinocytes and dermal fibroblasts against oxidative stress and identification of the bioactive phenolics. Biosci Biotechnol Biochem. 2013;77: 1682-8.

18. Agrawal M. Natural polyphenols based new therapeutic avenues for advanced biomedical applications. Drug Metab Rev. 2015;47:420-30.

19. Im WK, Park HJ, Lee KS, Lee JH, Kim YD, Kim K-H, Park S-J, Hong S, Jeon SH. Fisetin-rich extracts of Rhus verniciflua Stokes improve blood flow rates in mice fed both normal and high-fat diets. J Med Food. 2016;19:120-6.

20. Chen H, Wang C, Zhou H, Tao R, Ye J, Li W. Antioxidant capacity and identification of the constituents of ethyl acetate fraction from Rhus verniciflua Stokes by HPLC-MS. Nat Prod Res. 2017:31:1573-7.

21. Cheon SH, Kim KS, Kim S, Jung HS, Choi WC, Eo WK. Efficacy and safety of Rhus verniciflua stokes extracts in patients with previously treated advanced non-small cell lung cancer. Complement Med Res. 2011;18:77-83.
Ready to submit your research? Choose BMC and benefit from:

- fast, convenient online submission

- thorough peer review by experienced researchers in your field

- rapid publication on acceptance

- support for research data, including large and complex data types

- gold Open Access which fosters wider collaboration and increased citations

- maximum visibility for your research: over $100 \mathrm{M}$ website views per year

At $\mathrm{BMC}$, research is always in progress.

Learn more biomedcentral.com/submissions 